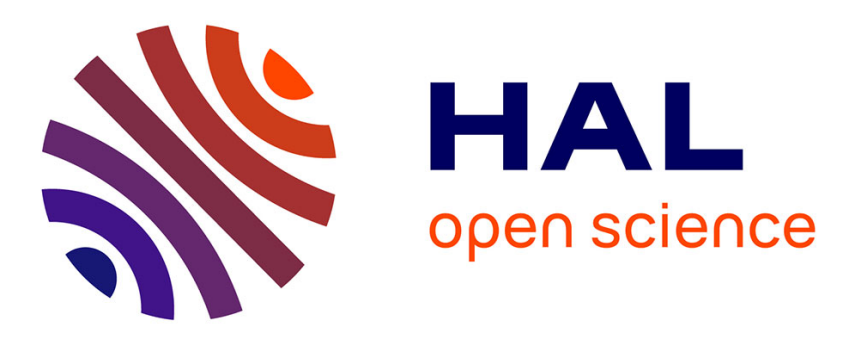

\title{
Organelle targeting during bacterial infection: insights from Listeria
}

\author{
Alice Lebreton, Fabrizia Stavru, Pascale Cossart
}

\section{To cite this version:}

Alice Lebreton, Fabrizia Stavru, Pascale Cossart. Organelle targeting during bacterial infection: insights from Listeria. Trends in Cell Biology, 2015, 25 (6), pp.330-8. 10.1016/j.tcb.2015.01.003 . pasteur-01162370

\section{HAL Id: pasteur-01162370}

\section{https://hal-pasteur.archives-ouvertes.fr/pasteur-01162370}

Submitted on 28 Aug 2017

HAL is a multi-disciplinary open access archive for the deposit and dissemination of scientific research documents, whether they are published or not. The documents may come from teaching and research institutions in France or abroad, or from public or private research centers.
L'archive ouverte pluridisciplinaire $\mathbf{H A L}$, est destinée au dépôt et à la diffusion de documents scientifiques de niveau recherche, publiés ou non, émanant des établissements d'enseignement et de recherche français ou étrangers, des laboratoires publics ou privés.

\section{다)(1) $(5$}

Distributed under a Creative Commons Attribution - NonCommerciall 4.0 International 


\title{
Organelle targeting during bacterial infection: Insights from Listeria
}

\author{
Alice Lebreton $^{1,2,3 \dagger}$, Fabrizia Stavru ${ }^{1,2,3}$ and Pascale Cossart ${ }^{1,2,3^{*}}$
}

1. Institut Pasteur, Unité des Interactions Bactéries-Cellules, 75015 Paris, France.

2. Institut National de la Santé et de la Recherche Médicale (Inserm), U604, 75015 Paris, France.

3. Institut National de la Recherche Agronomique (INRA,) USC2020, 75015 Paris France.

† Present address: École Normale Supérieure, Institut de Biologie de l'ENS (IBENS), Inserm U1024 and CNRS UMR 8197, 75005 Paris, France.

* Corresponding author: Cossart P. (pascale.cossart@pasteur.fr).

This is an accepted manuscript of an article published by Elsevier in Trends in Cell Biology in June 2015, available online as doi:10.1016/j.tcb.2015.01.003

Keywords: Listeria monocytogenes, cellular microbiology, organelle, mitochondria, nucleus, endomembrane system.

\begin{abstract}
Listeria monocytogenes, a facultative intracellular bacterium responsible for severe foodborne infections, is now recognized as a multifaceted model in infection biology. Comprehensive studies of the molecular and cellular basis of the infection have unravelled how the bacterium crosses the intestinal and feto-placental barriers, invades several cell types in which it multiplies and moves, and spreads from cell to cell. Interestingly, although Listeria does not actively invade host cell organelles, it can interfere with their function. We discuss the effect of Listeria on the ER and the mechanisms leading to the fragmentation of the mitochondrial network and its consequences, and review the strategies used by Listeria to subvert nuclear functions, more precisely to control host gene expression at the chromatin level.
\end{abstract}

\section{Highlights}

- Organelle manipulation by Listeria determines the outcome of infection.

- Disruption of mitochondrial dynamics affects the efficiency of Listeria infection.

- Bacteria secrete nucleomodulins to reprogram host cell transcription.

- Listeria-induced perturbations in ion homeostasis impact all organelles. 


\section{Bacterial life in the complex intracellular environment}

Intracellular bacteria have developed various strategies to invade, survive, and multiply inside cells. Many bacterial pathogens including Legionella, Chlamydia, Salmonella and Brucella co-opt inner membranes to create a specific compartment adapted to their needs [reviewed in 1,2]. Few bacteria, most of which are endosymbionts, reside inside pre-existing organelles [e.g. 3-5]. By contrast, several pathogenic species such as Listeria monocytogenes, Shigella flexneri and members of the genus Rickettsia, escape from their internalization vacuoles and complete their life cycle in the cytosol [reviewed in 6]. The respective benefits and detriments of these diverse habitats concerning nutrient availability or sensitivity to host immune recognition are difficult to compare. In each case, bacteria have evolved survival strategies adapted to their specific lifestyles. Despite of their different residence sites, most intracellular bacteria directly or indirectly interact with organelles and perturb their function. We illustrate this issue with the example of Listeria monocytogenes.

Organelles are among the most prominent features that distinguish eukaryotic from prokaryotic cells (see Glossary). They form intracellular compartments delimited by at least one membrane. They are defined by a characteristic composition and specific metabolic pathways. Organelles can furthermore act as platforms for signalling pathways, allowing the coordination of activities such as metabolism, innate immune sensing, and the stress response $[7,8]$. Therefore, they represent attractive targets for bacterial pathogens to hijack host cell function and ensure their own survival.

L. monocytogenes (hereafter also Listeria) is a food-borne bacterial pathogen capable of invasion and replication in phagocytic and non-phagocytic cells. This capacity allows it to cross the protective epithelial barriers of the human body and cause severe disease in immunocompromised individuals [reviewed in 9,10]. To invade host cells, Listeria expresses several dedicated proteins, notably internalin (InlA) and InlB. When the bacterium contacts the host cell membrane, InlA and InlB bind to E-Cadherin and to the hepatocyte growth factor (HGF) receptor Met respectively and induce bacterial internalization [reviewed in 11]. An internalization vacuole is formed from which the bacterium usually escapes; however, in vivo analysis of the crossing of the intestinal barrier has shown that Listeria can also transcytose across epithelial cells [12].

To evade membrane-bound structures, Listeria secretes two phospholipases (PlcA and $\mathrm{PlcB}$ ), as well as a cholesterol-dependent cytolysin, listeriolysin O (LLO) [13-15]. When the $\mathrm{pH}$ of the internalization vacuole acidifies, LLO forms pores in the vacuolar membrane and destabilizes it. In addition to its role in vacuole escape, LLO acts at several steps of the infection cycle and appears as a multifunctional toxin [see 16 for a recent review].

An alternative pathway implicating the intravacuolar replication of Listeria has been documented in macrophages of mice with severe combined immunodeficiency [17]. The formation of non-acidic, non-degradative compartments named spacious Listeria-containing phagosomes (SLAPS) is dependent on macroautophagy, and on moderate secretion of LLO. SLAPs have been proposed to provide a niche for persistent Listeria infection within macrophages, but do not represent a general rule for intracellular proliferation.

Listeria does not only escape the internalisation vacuole, but also avoids autophagic recognition, entrapment in autophagosomes and degradation. During cellular infection, the membrane damage induced by LLO leads to a transient amino-acid starvation, to which the host cell responds through inhibition of the mTOR pathway and induction of autophagy $[18,19]$. However, PlcA and PlcB inhibit the cell autophagic flux; by stalling pre- 
autophagosome structures, they protect bacteria from clearance [19]. Another player in escape from autophagy is the Listeria surface protein ActA, which recruits the actin polymerisation machinery onto the bacterial surface to promote intracellular movement and spreading to neighbouring cells [20,21]. The recruitment of actin by ActA [22] and that of the Major Vault Protein (MVP) by InlK [23] cover the bacterial surface and prevent the sensing and targeting of bacteria to autophagy [reviewed in 24].

Apart from its ability to escape from internalisation compartments and autophagosomes, Listeria has developed a broad range of strategies allowing it to interfere with the function of bona fide cellular organelles, i.e. pre-existing the bacterial infection. These activities range from the perturbation of vesicle trafficking, or of the ER, to more elaborate mechanisms leading to fragmentation of the mitochondrial network, or to the modulation of host gene expression via direct chromatin-targeting mechanisms. We review here our current understanding of these processes and of the benefit they convey to the bacterium.

\section{Perturbation of the endomembrane system during Listeria infection}

The endomembrane system defines the subset of cellular compartments enclosed by membrane structures that are involved in transport. It includes the ER, the Golgi apparatus, lysosomes, endo- and exocytic compartments, as well as the nuclear envelope. The different components of these systems can exchange membrane and contents through vesicular transport. During infection, Listeria interacts successively with several of these compartments.

\section{Inhibition of phagosomal killing}

A primary strategy of the cell to counteract invading bacteria is maturation and then fusion of the phagocytic vacuole containing the bacteria (the phagosome) with lysosomes, a pathway that is particularly active in macrophages (Fig. 1). The small GTPase Rab5a is associated with the membrane of early endosomes and phagosomes and recruits effectors required for phagosome maturation, including enzymes leading to phosphoinositide conversion $[25,26]$. Rab5a has also been reported to play a role in the killing of Listeria after uptake by macrophages, by inducing the translocation of the small Rho GTPase Rac to the phagosome, which in turn promotes the assembly, at the phago-lysosomal membrane, of a NADPH oxidase complex producing reactive oxygen species (ROS) in the mature phagosome [27]. By targeting Rab5a, Listeria blocks the maturation of phagosomes and thus avoids killing. Towards this aim, Listeria secretes a $40-\mathrm{kDa}$ protein annotated as a glyceraldehyde-3phosphate dehydrogenase (Lmo2459). Lmo2459 can mediate the ADP-ribosylation of Rab5a, which impedes phago-lysosomal maturation [28]; however, how Lmo2459 reaches its target Rab5a has not been defined. Whether a similar scenario occurs in epithelial cells is also unknown.

\section{Inhibition of lysosome fusion}

In addition to the mechanism described above, the pore-forming activity of LLO contributes to impede the fusion of lysosomes with the endocytic vacuole [29,30] (Fig. 1). Before bacterial escape into the cytosol, LLO-mediated perforation of the phagosomal membrane allows ion fluxes between compartments, with a notable drop in calcium concentration and an increase in $\mathrm{pH}$ inside the phagosome. This perturbation of the ion gradient across the phagosomal membrane inhibits fusion with LAMP1-positive lysosomes, 
as previously reported for the depletion of vacuolar calcium [31]. One should note that the activity of LLO itself is sensitive, in addition to $\mathrm{pH}$ variations [32], to other vacuolar ion concentrations, such as $\mathrm{K}^{+} / \mathrm{Cl}^{-}$[33]. In line with this, the host chloride channel Cystic Fibrosis Transmembrane Conductance Regulator (CFTR) located in the phagosomal membrane promotes Listeria phagosomal escape by increasing intraphagosomal $\mathrm{Cl}^{-}$concentration and thereby potentiating LLO oligomerization [33].

Altogether, by blocking the maturation of endocytic compartments, LLO and Lmo2459 allow the bacteria to avoid intraphagolysosomal degradation.

\section{Perturbation of intracellular calcium storage and signalling}

LLO triggers a massive increase in cytosolic calcium concentration, primarily due to an influx of extracellular $\mathrm{Ca}^{2+}$ via pores in the plasma membrane [34]. $\mathrm{Ca}^{2+}$ influx has been shown to promote bacterial entry, possibly through the fusion of subcortical vesicles that increase the membrane availability required for the uptake of a particle as large as a bacterium $[35,36]$. In addition, compartments of the endomembrane system, such as the ER, release stored $\mathrm{Ca}^{2+}$ into the cytosol upon infection. This release relies on two LLO-dependent mechanisms. First, InlB and LLO trigger signalling cascades including protein tyrosine kinases, which result in the activation of phospholipases C (PLC) and the generation of inositol triphosphate $\left(\mathrm{IP}_{3}\right)$, thereby stimulating the $\mathrm{IP}_{3}$ receptor $\mathrm{Ca}^{2+}$ channels $[37,38]$. Second, LLO is responsible for reversible damages to the ER and lysosome membranes and the release of their content [30,38], inducing a transient increase in cytosolic calcium concentration. In both epithelial and immune cells, $\mathrm{LLO}$-induced $\mathrm{Ca}^{2+}$ signals were shown to lead to a production of pro-inflammatory cytokines, which may contribute to pathology in different tissues, such as the intestine and the liver [38-40].

\section{Activation of the unfolded protein response/ER stress}

The ER damage mediated by LLO even prior to bacterial entry causes rapid swelling and loss of ER-tracker staining, which is typical of ER stress [38]. This stress is likely amplified by the concomitant increase in cytosolic calcium concentration $[38,41]$. In response to this stress, the unfolded protein response (UPR) is activated, and ultimately leads to cell death if the infection is sustained $[30,38,42]$. This cell response is considered to be antibacterial, as induction of the UPR prior to infection reduces the bacterial number [42].

Similar to the ER-based UPR, the mitochondrial UPR (mtUPR) is induced upon mitochondrial stress, where the protein ATFS1 accumulates in the nucleus instead of mitochondria and activates the transcription of proteins exerting protective functions in mitochondria $[38,43]$. The mtUPR pathway was recently found to activate an innate immunity program, suggesting that surveillance of organelle homeostasis is part of the active antimicrobial strategy in infected cells [44]. It remains to be uncovered whether this pathway is activated upon Listeria infection.

\section{Mitochondrial dynamics and infection}

Mitochondria are essential organelles that form a dynamic network within the cell. They play a crucial role in energy metabolism and cellular signalling pathways such as innate immunity, calcium signalling or apoptosis, which makes them an attractive target for pathogens to interfere with cellular function. Indeed, several bacteria and viruses modulate mitochondrial function to maintain their replicative niche by preventing host cell apoptosis or 
to favour dissemination by inducing apoptosis [reviewed in 45-47]. A variety of physiological and pathological conditions have been shown to induce either fusion or fission of the mitochondrial network and thereby affect mitochondrial function.

Infection with L. monocytogenes induces the host cell mitochondrial network to undergo a rapid, transient fragmentation already at early time points of infection (1-3h), along with a loss in the mitochondrial membrane potential, which results in decreased intracellular ATP levels [48] (Fig. 2A). This effect is specific to pathogenic Listeria, as it is not observed with the non-pathogenic species $L$. innocua or several other intracellular bacteria. The main bacterial factor responsible for mitochondrial fragmentation is the pore-forming toxin LLO, which causes calcium influx across the plasma membrane, although the exact signalling pathway leading to mitochondrial fragmentation is yet to be discovered. Other pore-forming toxins from the same family as LLO, such as pneumolysin, perfringolysin or streptolysin induce the same morphological phenotype as LLO, but it is largely unknown whether and how infection with the corresponding bacteria would affect mitochondria. In contrast to the bacterial pore-forming proteins Helicobacter pylori VacA, Neisseria gonorrhoeae PorB or Staphylococcus aureus Panton-Valentine leukocydin [reviewed in 46] (Fig 2B), which reach and perturb mitochondrial membranes, LLO does not appear to localize to mitochondria, although it might do so if delivered by bacterial membrane vesicles, as has been recently shown for the enteropathogenic Escherichia coli Hly toxin [49].

Strikingly, functional mitochondrial dynamics appears to be important for Listeria infection, because perturbing the fusion-fission balance by siRNA against key components of the fusion machinery causes a decrease in the infection efficiency, while interfering with fission results in its augmentation. Together, these findings led to the hypothesis that Listeria targets the host cell mitochondrial network to temporarily shut down cellular bioenergetics and to interfere with mitochondrial innate immune signalling [48]. Current efforts are aimed at obtaining a global view of the mitochondrial pathways that are reorganized upon Listeria infection (Box 1).

Mechanistically, the atypical mitochondrial fragmentation induced by Listeria differs from VacA-induced mitochondrial fragmentation [50], since it is independent of the key mitochondrial fission protein, the dynamin-related protein Drp1, which dissociates from mitochondria upon LLO treatment [51]. As in canonical fragmentation, the ER and actin were found to contribute significantly to the process; however, the fragmentation sites were marked by the ER also in the absence of Drp1, suggesting that although the initial constriction machinery is shared, molecules other than Drp1 can terminate mitochondrial abscission downstream of the ER. Uncovering such molecule(s) is a challenging issue, since to date LLO and Listeria are the only known exogenous stimuli of Drp1-independent mitochondrial fragmentation (Box 1).

Recently, mitochondria have been shown to play an important role in innate immunity through Mitochondrial AntiViral Signalling protein (MAVS), an adaptor protein located on the mitochondrial outer membrane that regulates signal transduction from cytosolic RNA sensors. MAVS activity has been shown to depend on mitochondrial dynamics and function [52-54]. Through the assembly of a large signalling complex on mitochondria, activated MAVS triggers nuclear translocation of the transcription factors NF- $\kappa B$, IRF3 and IRF7, which results in the production of interferons, cytokines and interferon-stimulated genes (ISGs) [reviewed in 55]. Similar to other mitochondrial tail-anchored proteins, MAVS has additionally been found to localize at peroxisomes [56]. MAVS is one of the mediators of the innate immune response to Listeria in epithelial cells, leading to the production of type I and type III interferons $[57,58]$. Consistent with the disruption of mitochondrial morphology and 
function by Listeria, it appears that MAVS-dependent stimulation of the interferon response is mainly carried out by peroxisomal rather than mitochondrial MAVS [58].

\section{Targeting nuclear functions: strategies to reprogram the host}

Upon infection by Listeria, the transcriptome of host cells is vastly reshaped. Different patterns of transcriptional response have been identified, depending on the nature of target cells in experiments performed in vitro [59-62], or on more complex regulatory circuits occurring in mouse models $[63,64]$. This reprogramming is largely the consequence of cellular signalling events occurring upon bacterial entry and/or subsequent recognition of Pathogen Associated Molecular Patterns (PAMPs) by a variety of host sensors named PatternRecognition Receptors (PRRs) [reviewed in 65]. In addition to these pathways, the notion recently emerged that pathogenic bacteria could manipulate host transcription by directly acting at the nuclear level and interfering with chromatin-based regulation $[66,67]$, giving rise to the emerging field of patho-epigenetics (Box 1). Research on Listeria has been on the leading edge of this topic. As documented below, it has revealed that bacterial-triggered signalling events can impact the post-translational modification of histone tails [68], and that a virulence factor, belonging to a new class of bacterial secreted effectors named nucleomodulins [69] can localize to the cell nucleus and perturb chromatin silencing.

\section{Perturbation of histone modifications}

Eukaryotic DNA is organised in the nucleus into a structure named chromatin [reviewed in 70]. The primary unit of chromatin architecture is the nucleosome, which consists of a core octamer of histones $\mathrm{H} 2 \mathrm{~A} / \mathrm{B}, \mathrm{H} 3$ and $\mathrm{H} 4$, around which DNA wraps together with linker histones H1. Successive nucleosomes are packed more or less tightly to form chromatin fibres. The post-translational status of core histone tails plays a key function in the organization of this structure; by modulating the compaction of the chromatin assembly, and acting as specific docking sites for regulatory factors, these modifications constitute a histone code that adds another layer in the regulation of gene expression and cell fate decisions [71].

\section{LLO induces histone deacetylation and dephosphorylation}

Listeria was one of the first examples of an invasive bacterium reported to have an effect on its host's histone modifications [72]. Following the infection of HeLa cells, the total levels of serine 10 phosphorylation histone $\mathrm{H} 3$ (H3S10deP) and lysine acetylation of histone $\mathrm{H} 3$ (H3S10deP), as well as acetylation of histone H4 (H4KdeAc) are significantly decreased (Fig. 3, left). Both H3S10deP and H4KdeAc are induced by secreted LLO, and can be recapitulated by addition of the purified toxin. Transcriptional studies have highlighted that a specific subset of host genes is de-regulated within 20 minutes of LLO treatment. Interestingly, Chromatin Immunoprecipitation (ChIP) experiments have revealed that H3S10deP and $\mathrm{H} 4 \mathrm{KdeAc}$ occur specifically at promoter genes that are differentially regulated upon LLO addition, suggesting that histone modifications induced by LLO are responsible for the reprogramming of host gene expression. In terms of mechanism, the intracellular signalling leading to $\mathrm{H} 3 \mathrm{~S} 10 \mathrm{deP}$ is dependent on potassium efflux driven by increased membrane permeability upon LLO treatment, and can be recapitulated with other bacterial pore-forming toxins or potassium-specific ionophores [73]. Future investigations are needed to clarify how the decrease in cellular potassium concentration is sensed, and which signalling pathways result in histone modifications. 


\section{Histone deacetylation by SIRT2}

In contrast to H3S10deP and $\mathrm{H} 4 \mathrm{KdeAc}, \mathrm{H} 3 \mathrm{KdeAc}$ has been shown to be a LLOindependent process [72]. It is driven by another virulence factor, InlB, one of the two surface internalins involved in Listeria entry into its target cells [74]. Upon binding to its cell membrane receptor Met (the receptor of HGF), InlB triggers the activation of a signalling cascade via the PI3K/Akt pathway, which results in the translocation of the tubulindeacetylase SIRT2 [75] from the cytoplasm to the chromatin fraction of the nucleus (Fig. 3, right). There, SIRT2 is responsible for the specific deacetylation of lysine 18 of $\mathrm{H} 3$ during infection, and for the transcriptional deregulation of hundreds of host genes. ChIP experiments showed that the promoter of genes that are repressed in a SIRT2-dependent manner during infection are enriched in deacetylated H3K18, suggesting that the activity of the histone deacetylase at these promoters is responsible for their repression during infection. The subversion of SIRT2 function by InlB proves to be pro-bacterial, as SIRT2 ${ }^{--}$mice can control listeriosis more efficiently than their wild type littermates. Further studies will be required to identify which SIRT2-regulated genes are most important for host defence against Listeria. Given that Listeria is the only known stimulus that induces SIRT2 translocation to the nucleus, it will provide an essential tool to elucidate the precise mechanisms mediating SIRT2 regulation at the chromatin.

\section{Interaction of the nucleomodulin LntA with the heterochromatin protein BAHD1}

Upon infection of epithelial cells, Listeria, like other intracellular bacteria, activates host innate immunes responses, which lead to the production and secretion of a variety of cytokines including type I and III interferons [reviewed in 76]. However, the expression of downstream ISGs is repressed by the heterochromatinization factor, BAHD1 [77] (Fig. 3, bottom). In non-infected cells, BAHD1 can nucleate the formation of hererochromatin at the promoter of a subset of host genes [78]; during infection, BAHD1 additionally targets ISGs via a so-far unknown mechanism. Listeria can, under conditions that are still poorly defined, secrete the nucleomodulin LntA into the cell cytosol. This protein enters the host nucleus where it binds directly to BAHD1, causing it to dissociate from chromatin, and thus activating the expression of ISGs [77,79]. The lntA virulence gene, and a tight regulation of its expression, is required for an optimal infectious process in vivo, indicating that the deregulation of BAHD1 must be carefully timed and tuned. In line with this hypothesis, hypomorphic $\mathrm{BAHD}^{+/}{ }^{-}$mice are more resistant to Listeria infection than their wild type littermates. The precise effects of the host interferon responses on the patho-physiological outcomes of infection is still a matter of debate [80]; their study will certainly lead to surprising findings, which should clarify why their accurate modulation is essential to the host as well as to the bacterium.

Other secreted effectors of Listeria that can translocate to the host cell nucleus are currently under investigation. Their extensive functional characterisation should highlight novel mechanisms of subversion of nuclear functions by Listeria nucleomodulins.

\section{Concluding remarks}

The strategies of organelle subversion by Listeria that we have reviewed here reveal some of the ingenious tricks allowing the bacterium to adapt to its intracellular environment. Via a variety of molecular mechanisms, the bacterium is able to reorganize the host cell endomembrane dynamics, its energetics and gene expression program, most often for its own 
profit. Depending on the organelle, strategies and consequences differ significantly. For instance, mitochondrial targeting results in a global reshaping of their structure, dynamics and functionality, and we are only beginning to understand the functional consequences of these events; they might represent the mammalian counterpart to the surveillance pathway described in Caenorhabditis elegans, which correlates mitochondrial dysfunction with pathogen attack [81].

In contrast to the profound effect on mitochondria, the harnessing of chromatin by the Listeria protein LntA appears as a more focused process. It allows Listeria to selectively control the transcription of a subset of host genes, like ISGs, independently from other nuclear functions affected by infection, such as the DNA damage response described in other studies $[82,83]$.

Organelle targeting during infection is not restricted to L. monocytogenes; a number of viruses, parasites and intracellular bacteria seem to have evolved alternative approaches to hijack the functions of metazoan organelles such as mitochondria or nuclei [see for instance 84-87], of which nuclear targeting has been previously reviewed [69]. As exemplified by $H$. pylori VacA [88] or the enteropathogenic E. coli proteins EspF and Map [89,90], targeting of microbial proteins to mitochondria can directly or indirectly disrupt the mitochondrial membranes and modulate cell death. Recently, more subtle mechanisms have emerged: the $V$. cholerae secreted effector VopE acts as a mitochondria-localized GTPase-activating protein for the mitochondrial protein Miro, thereby disrupting its ability to mediate kinesin-dependent mitochondrial motility and resulting in mitochondrial fragmentation [91]. Similar strategies are used by plant pathogens. For instance, the type III secretion system (T3SS) of Pseudomonas syringae pv tomato DC3000 secretes Hop1N, a cysteine protease targeted to the host chloroplast, which interferes with photosynthesis by degrading a component of the photosystem, PsbQ [92]. This suppresses ROS production involved in the plant innate immune response to bacterial infection, and counteracts host programmed cell death. Other pathogens, such as Chlamydia, Legionella or Toxoplasma recruit mitochondria to manipulate bioenergetic and innate immune pathways [93-95]. In the case of Toxoplasma, the molecular basis for this phenomenon has recently been uncovered with the identification of mitochondrial association factor 1 (MAF1), a Toxoplasma protein that directly binds host cell mitochondria [96].

Ultimately, the subversion of organelles can result in their colonisation, as mentioned in the introduction. This has so far been essentially documented for endosymbionts, such as the bacterial inhabitants of paramecium macro- and micronuclei [97] or the intra-mitochondrial bacterial species discovered in ticks, Candidatus Midichloria mitochondrii [3], and intranuclear growth has also been reported for a pathogen, the spotted fever agent Rickettsia rickettsii $[98,99]$.

Together, these studies pave the way to a better understanding of how bacteria take advantage of their host at the nano- and microscopic levels. However, our perception of organelle dynamics during infection remains incomplete. Functional studies by high resolution and live-cell imaging should, in the coming years, allow us to better define the mechanisms that govern the morphological and functional changes in the ER and mitochondria. They should allow us to fully appreciate the impact of bacteria on vesicle trafficking, and the key role played by the cytoskeleton in the redistribution of intracellular compartments during infection. Whether Listeria infection also influences other key organelles such as peroxisomes, the nucleo-cytoplasmic transport pathways, or the architecture and function of sub-compartments not delineated by a membrane, such as Pbodies or stress granules, are still open questions. 


\section{Acknowledgements}

We are grateful to Mélanie Hamon and Javier Pizarro-Cerdá for critical reading of the manuscript.

\section{Role of the funding sources}

Work in the Cossart laboratory received financial support from Pasteur Institute, INSERM and INRA, from the European Research Council (ERC) advanced Grant 233348, from Agence Nationale de la Recherche (ANR blancs Mitopatho 12-BSV3-0017-03 and EpiLis 11BSV3-003-01; ERA-NET Infect-ERA ProAntiLis), from Laboratoires d'Excellence (IBEID ANR-10- LABX-62-01), from Fondation Jeantet and Fondation le Roch Les Mousquetaires. P. Cossart is a Senior International Research Scholar of the Howard Hughes Medical Institute, F. Stavru is a CNRS research associate.

We declare no conflict of interest.

\section{References}

1 Salcedo, S.P. and Holden, D.W. (2005) Bacterial interactions with the eukaryotic secretory pathway. Curr. Opin. Microbiol. 8, 92-98

2 Cossart, P. and Maloy, S.R. (2014) Bacterial Pathogenesis,

3 Sassera, D. et al. (2006) "Candidatus Midichloria mitochondrii," an endosymbiont of the tick Ixodes ricinus with a unique intramitochondrial lifestyle. Int J Syst Evol Microbiol 56, 2535-2540

4 Fujishima, M. and Kodama, Y. (2012) Endosymbionts in paramecium. Eur. J. Protistol. 48, 124-137

5 Schulz, F. et al. (2014) Life in an unusual intracellular niche: a bacterial symbiont infecting the nucleus of amoebae. ISME J 8, 1634-1644

6 Kay, S. et al. (2007) A bacterial effector acts as a plant transcription factor and induces a cell size regulator. Science 318, 648-651

7 Malhas, A. et al. (2011) The nucleoplasmic reticulum: form and function. Trends Cell Biol 21, 362-373

8 Tait, S.W.G. and Green, D.R. (2012) Mitochondria and cell signalling. J Cell Sci 125, 807-815

9 Allerberger, F. and Wagner, M. (2010) Listeriosis: a resurgent foodborne infection. Clin. Microbiol. Infect. 16, 16-23

10 Cossart, P. (2011) Illuminating the landscape of host-pathogen interactions with the bacterium Listeria monocytogenes. Proc Natl Acad Sci USA 108, 19484-19491

11 Pizarro-Cerda, J. et al. (2012) Entry of Listeria monocytogenes in mammalian epithelial cells: an updated view. Cold Spring Harb Perspect Med 2,

12 Nikitas, G. et al. (2011) Transcytosis of Listeria monocytogenes across the intestinal barrier upon specific targeting of goblet cell accessible E-cadherin. J Exp Med 208, 2263-2277

13 Gaillard, J.L. et al. (1987) In vitro model of penetration and intracellular growth of Listeria monocytogenes in the human enterocyte-like cell line Caco-2. Infect Immun 55, 2822-2829

14 Gründling, A. et al. (2003) Requirement of the Listeria monocytogenes broad-range phospholipase PCPLC during infection of human epithelial cells. J Bacteriol 185, 6295-6307

15 Alberti-Segui, C. et al. (2007) Differential function of Listeria monocytogenes listeriolysin O and phospholipases C in vacuolar dissolution following cell-to-cell spread. Cell Microbiol 9, 179-195

16 Hamon, M.A. et al. (2012) Listeriolysin O: the Swiss army knife of Listeria. Trends Microbiol 20, 360368

17 Birmingham, C.L. et al. (2008) Listeriolysin O allows Listeria monocytogenes replication in macrophage vacuoles. Nature 451, 350-354

18 Meyer-Morse, N. et al. (2010) Listeriolysin O is necessary and sufficient to induce autophagy during Listeria monocytogenes infection. PLoS ONE 5, e8610

19 Tattoli, I. et al. (2013) Listeria phospholipases subvert host autophagic defenses by stalling preautophagosomal structures. EMBO J 32, 3066-3078

20 Tilney, L.G. and Portnoy, D.A. (1989) Actin filaments and the growth, movement, and spread of the 
intracellular bacterial parasite, Listeria monocytogenes. J Cell Biol 109, 1597-1608

21 Kocks, C. et al. (1992) L. monocytogenes-induced actin assembly requires the actA gene product, a surface protein. Cell 68, 521-531

22 Yoshikawa, Y. et al. (2009) Listeria monocytogenes ActA-mediated escape from autophagic recognition. Nat Cell Biol 11, 1233-1240

23 Dortet, L. et al. (2011) Recruitment of the Major Vault Protein by InlK: A Listeria monocytogenes Strategy to Avoid Autophagy. PLoS Pathog 7, e1002168

24 Dortet, L. et al. (2012) Listeria and autophagy escape: involvement of InlK, an internalin-like protein. Autophagy 8, 132-134

25 Wandinger-Ness, A. and Zerial, M. (2014) Rab proteins and the compartmentalization of the endosomal system. Cold Spring Harb Perspect Biol 6, a022616

26 Pizarro-Cerda, J. et al. (2014) Phosphoinositides and host-pathogen interactions. Biochim Biophys Acta DOI: 10.1016/j.bbalip.2014.09.011

27 Prada-Delgado, A. et al. (2001) Interferon-gamma listericidal action is mediated by novel Rab5a functions at the phagosomal environment. J Biol Chem 276, 19059-19065

28 Alvarez-Domínguez, C. et al. (2008) Characterization of a Listeria monocytogenes protein interfering with Rab5a. Traffic 9, 325-337

29 Henry, R. et al. (2006) Cytolysin-dependent delay of vacuole maturation in macrophages infected with Listeria monocytogenes. Cell Microbiol 8, 107-119

30 Shaughnessy, L.M. et al. (2006) Membrane perforations inhibit lysosome fusion by altering pH and calcium in Listeria monocytogenes vacuoles. Cell Microbiol 8, 781-792

31 Pryor, P.R. et al. (2000) The role of intraorganellar $\mathrm{Ca}^{2+}$ in late endosome-lysosome heterotypic fusion and in the reformation of lysosomes from hybrid organelles. J Cell Biol 149, 1053-1062

32 Beauregard, K.E. et al. (1997) pH-dependent perforation of macrophage phagosomes by listeriolysin O from Listeria monocytogenes. J Exp Med 186, 1159-1163

33 Radtke, A.L. et al. (2011) Listeria monocytogenes exploits cystic fibrosis transmembrane conductance regulator (CFTR) to escape the phagosome. Proc Natl Acad Sci USA 108, 1633-1638

34 Repp, H. et al. (2002) Listeriolysin of Listeria monocytogenes forms $\mathrm{Ca}^{2+}$-permeable pores leading to intracellular $\mathrm{Ca}^{2+}$ oscillations. Cell Microbiol 4, 483-491

35 Dramsi, S. and Cossart, P. (2003) Listeriolysin O-mediated calcium influx potentiates entry of Listeria monocytogenes into the human Hep-2 epithelial cell line. Infect Immun 71, 3614-3618

36 Vadia, S. and Seveau, S. (2014) Fluxes of $\mathrm{Ca}^{2+}$ and $\mathrm{K}^{+}$are required for the listeriolysin O-dependent internalization pathway of Listeria monocytogenes. Infect Immun 82, 1084-1091

37 Bierne, H. et al. (2000) The invasion protein InIB from Listeria monocytogenes activates PLC-gamma1 downstream from PI 3-kinase. Cell Microbiol 2, 465-476

38 Gekara, N.O. et al. (2007) The multiple mechanisms of $\mathrm{Ca}^{2+}$ signalling by listeriolysin O, the cholesteroldependent cytolysin of Listeria monocytogenes. Cell Microbiol 9, 2008-2021

39 Dewamitta, S.R. et al. (2010) Listeriolysin O-dependent bacterial entry into the cytoplasm is required for calpain activation and interleukin-1 alpha secretion in macrophages infected with Listeria monocytogenes. Infect Immun 78, 1884-1894

40 Tsuchiya, K. et al. (2010) Involvement of absent in melanoma 2 in inflammasome activation in macrophages infected with Listeria monocytogenes. J Immunol 185, 1186-1195

41 Cywes Bentley, C. et al. (2005) Extracellular group A Streptococcus induces keratinocyte apoptosis by dysregulating calcium signalling. Cell Microbiol 7, 945-955

42 Pillich, H. et al. (2012) Activation of the unfolded protein response by Listeria monocytogenes. Cell Microbiol 14, 949-964

43 Haynes, C.M. et al. (2013) Evaluating and responding to mitochondrial dysfunction: the mitochondrial unfolded-protein response and beyond. Trends Cell Biol 23, 311-318

44 Pellegrino, M.W. et al. (2014) Mitochondrial UPR-regulated innate immunity provides resistance to pathogen infection. Nature 516, 414-417

45 Galluzzi, L. et al. (2008) Viral control of mitochondrial apoptosis. PLoS Pathog 4, e1000018

46 Rudel, T. et al. (2010) Interactions between bacterial pathogens and mitochondrial cell death pathways. Nat Rev Microbiol 8, 693-705

47 Ashida, H. et al. (2011) Cell death and infection: a double-edged sword for host and pathogen survival. $J$ Cell Biol 195, 931-942

48 Stavru, F. et al. (2011) Listeria monocytogenes transiently alters mitochondrial dynamics during infection. Proc Natl Acad Sci USA 108, 3612-3617

49 Bielaszewska, M. et al. (2013) Enterohemorrhagic Escherichia coli hemolysin employs outer membrane 
vesicles to target mitochondria and cause endothelial and epithelial apoptosis. PLoS Pathog 9, e1003797

50 Jain, P. et al. (2011) Helicobacter pylori vacuolating cytotoxin A (VacA) engages the mitochondrial fission machinery to induce host cell death. Proc Natl Acad Sci USA 108, 16032-16037

51 Stavru, F. et al. (2013) Atypical mitochondrial fission upon bacterial infection. Proc Natl Acad Sci USA $110,16003-16008$

52 Castanier, C. and Arnoult, D. (2011) Mitochondrial localization of viral proteins as a means to subvert host defense. Biochim Biophys Acta 1813, 575-583

53 Onoguchi, K. et al. (2010) Virus-infection or 5'ppp-RNA activates antiviral signal through redistribution of IPS-1 mediated by MFN1. PLoS Pathog 6, e1001012

54 Koshiba, T. et al. (2011) Mitochondrial membrane potential is required for MAVS-mediated antiviral signaling. Sci Signal 4, ra7-ra7

55 West, A.P. et al. (2011) Mitochondria in innate immune responses. Nat Rev Immunol 11, 389-402

56 Dixit, E. et al. (2010) Peroxisomes are signaling platforms for antiviral innate immunity. Cell 141, 668681

57 Hagmann, C.A. et al. (2013) RIG-I detects triphosphorylated RNA of Listeria monocytogenes during infection in non-immune cells. PLOS ONE 8, e62872

58 Odendall, C. et al. (2014) Diverse intracellular pathogens activate type III interferon expression from peroxisomes. Nat Immunol 15, 717-726

59 Cohen, P. et al. (2000) Monitoring cellular responses to Listeria monocytogenes with oligonucleotide arrays. J Biol Chem 275, 11181-11190

60 Baldwin, D.N. et al. (2003) A gene-expression program reflecting the innate immune response of cultured intestinal epithelial cells to infection by Listeria monocytogenes. Genome Biol 4, R2

61 McCaffrey, R.L. et al. (2004) A specific gene expression program triggered by Gram-positive bacteria in the cytosol. Proc Natl Acad Sci USA 101, 11386-11391

62 Frande-Cabanes, E. et al. (2014) Dissociation of innate immune responses in microglia infected with Listeria monocytogenes. Glia 62, 233-246

63 Lecuit, M. et al. (2007) Functional genomic studies of the intestinal response to a foodborne enteropathogen in a humanized gnotobiotic mouse model. J Biol Chem 282, 15065-15072

64 Archambaud, C. et al. (2012) Impact of lactobacilli on orally acquired listeriosis. Proc Natl Acad Sci USA $109,16684-16689$

65 Eitel, J. et al. (2010) Innate immune recognition and inflammasome activation in Listeria monocytogenes infection. Frontiers in Microbiology 1, 149

66 Bierne, H. et al. (2012) Epigenetics and bacterial infections. Cold Spring Harb Perspect Med 2, a010272-a010272

67 Minàrovits, J. (2009) Microbe-induced epigenetic alterations in host cells: the coming era of pathoepigenetics of microbial infections. A review. Acta Microbiol Immunol Hung 56, 1-19

68 Hamon, M.A. and Cossart, P. (2008) Histone modifications and chromatin remodeling during bacterial infections. Cell Host Microbe 4, 100-109

69 Bierne, H. and Cossart, P. (2012) When bacteria target the nucleus: the emerging family of nucleomodulins. Cell Microbiol 14, 622-633

70 Felsenfeld, G. and Groudine, M. (2003) Controlling the double helix. Nature 421, 448-453

71 Jenuwein, T. and Allis, C.D. (2001) Translating the histone code. Science 293, 1074-1080

72 Hamon, M.A. et al. (2007) Histone modifications induced by a family of bacterial toxins. Proc Natl Acad Sci USA 104, 13467-13472

73 Hamon, M.A. and Cossart, P. (2011) $\mathrm{K}^{+}$efflux is required for histone H3 dephosphorylation by Listeria monocytogenes listeriolysin $\mathrm{O}$ and other pore-forming toxins. Infect Immun 79, 2839-2846

74 Eskandarian, H.A. et al. (2013) A role for SIRT2-dependent histone H3K18 deacetylation in bacterial infection. Science 341, 1238858

75 North, B.J. et al. (2003) The human Sir2 ortholog, SIRT2, is an NAD+-dependent tubulin deacetylase. Mol Cell 11,437-444

76 Dussurget, O. et al. (2014) The bacterial pathogen Listeria monocytogenes and the interferon family: type I, type II and type III interferons. Front Cell Infect Microbiol 4, 50

77 Lebreton, A. et al. (2011) A bacterial protein targets the BAHD1 chromatin complex to stimulate type III interferon response. Science 331, 1319-1321

78 Bierne, H. et al. (2009) Human BAHD1 promotes heterochromatic gene silencing. Proc Natl Acad Sci USA 106, 13826-13831

79 Lebreton, A. et al. (2014) Structural basis for the inhibition of the chromatin repressor BAHD1 by the bacterial nucleomodulin LntA. MBio 5, e00775-13 
80 Decker, T. et al. (2005) The yin and yang of type I interferon activity in bacterial infection. Nat Rev Immunol 5, 675-687

81 Liu, Y. et al. (2014) Caenorhabditis elegans pathways that surveil and defend mitochondria. Nature 508, 406-410

82 Leitão, E. et al. (2014) Listeria monocytogenes induces host DNA damage and delays the host cell cycle to promote infection. Cell Cycle 13,

83 Samba-Louaka, A. et al. (2014) Listeria monocytogenes Dampens the DNA Damage Response. PLoS Pathog 10, e1004470

84 Pennini, M.E. et al. (2010) Histone Methylation by NUE, a Novel Nuclear Effector of the Intracellular Pathogen Chlamydia trachomatis. PLoS Pathog 6, e1000995

85 Rolando, M. et al. (2013) Legionella pneumophila effector RomA uniquely modifies host chromatin to repress gene expression and promote intracellular bacterial replication. Cell Host Microbe 13, 395-405

86 Anand, S.K. and Tikoo, S.K. (2013) Viruses as modulators of mitochondrial functions. Adv Virol 2013, 738794

87 Yarbrough, M.L. et al. (2014) Viral subversion of nucleocytoplasmic trafficking. Traffic 15, 127-140

88 Galmiche, A. et al. (2000) The N-terminal $34 \mathrm{kDa}$ fragment of Helicobacter pylori vacuolating cytotoxin targets mitochondria and induces cytochrome c release. EMBO J 19,6361-6370

89 Kenny, B. and Jepson, M. (2000) Targeting of an enteropathogenic Escherichia coli (EPEC) effector protein to host mitochondria. Cell Microbiol 2, 579-590

90 Nougayrède, J.-P. and Donnenberg, M.S. (2004) Enteropathogenic Escherichia coli EspF is targeted to mitochondria and is required to initiate the mitochondrial death pathway. Cell Microbiol 6, 1097-1111

91 Suzuki, M. et al. (2014) Vibrio cholerae T3SS Effector VopE Modulates Mitochondrial Dynamics and Innate Immune Signaling by Targeting Miro GTPases. Cell Host Microbe 16, 581-591

92 Rodríguez-Herva, J.J. et al. (2012) A bacterial cysteine protease effector protein interferes with photosynthesis to suppress plant innate immune responses. Cell Microbiol 14, 669-681

93 Jones, T.C. and Hirsch, J.G. (1972) The interaction between Toxoplasma gondii and mammalian cells. II. The absence of lysosomal fusion with phagocytic vacuoles containing living parasites. J Exp Med 136, 1173-1194

94 Horwitz, M.A. (1983) Formation of a novel phagosome by the Legionnaires' disease bacterium (Legionella pneumophila) in human monocytes. J Exp Med 158, 1319-1331

95 Matsumoto, A. et al. (1991) Morphological studies of the association of mitochondria with chlamydial inclusions and the fusion of chlamydial inclusions. J Electron Microsc (Tokyo) 40, 356-363

96 Pernas, L. et al. (2014) Toxoplasma effector MAF1 mediates recruitment of host mitochondria and impacts the host response. PLoS Biol 12, e1001845

97 Görtz, H.D. and Wiemann, M. (1989) Route of infection of the bacteria Holospora elegans and Holospora obtusa into the nuclei of Paramecium caudatum. Eur. J. Protistol. 24, 101-109

98 Wolbach, S.B. (1919) Studies on Rocky Mountain spotted Fever. J Med Res 41, 1-198

99 Iwamasa, K. et al. (1992) Ultrastructural study of the response of cells infected in vitro with causative agent of spotted fever group rickettsiosis in Japan. APMIS 100, 535-542

100 Schrader, M. (2006) Shared components of mitochondrial and peroxisomal division. Biochim Biophys Acta 1763, 531-541

101 Schröder, M. (2008) Endoplasmic reticulum stress responses. Cell Mol Life Sci 65, 862-894 


\section{Box 1. Outstanding Questions}

1. Does the organelle response to infection impact bacterial replication beyond innate immune signalling? In particular, how does mitochondrial bioenergetics affect bacterial replication?

2. What molecules perform mitochondrial abscission in the absence of the main fission protein Drp1? Is this pathway also at work in Drp1-containing cells?

3. Does Listeria infection cause peroxisome restructuring, given that the mitochondrial division machinery also acts in peroxisomal division [100]?

4. Can cells keep an epigenetic memory of previous infections? The discovery that bacterial pathogens can change the pattern of histone modification on chromatin has led to the tempting hypothesis that infected cells could be imprinted with a memory of infection, independently of the well-described commitment of memory B and T lymphocytes. Research in the patho-epigenetics field still has to provide evidence that the epigenetic marks deposited during infection are long-lasting and play a role in host cell behaviour during re-infection.

5. Are the infection-induced signals from different organelles integrated? If they are, how is this achieved, and at which cellular level? 


\section{Glossary}

Chromatin: Eukaryotic DNA is condensed into an organised ribonucleoproteic assembly named chromatin. The more or less dense structure of this arrangement has several functions. During metaphase, chromatin displays its most compact form, which participates in the partition of genetic material among daughter cells. In other phases, the tightness of chromatin varies locally, on distances spanning over a few nucleosomes to a whole chromosome. The ability of chromatin to switch from an open (euchromatin) to a closed (heretochromatin) state is a potent regulator of gene expression.

Intracellular bacteria: Bacteria that invade and replicate inside host cells. They are subdivided into facultative intracellular bacteria (for example Listeria monocytogenes, Salmonella Typhimurium or Shigella flexneri) and obligate intracellular bacteria (for example several Rickettsia).

Mitochondrial membrane potential: An electrochemical gradient formed when the electron transport chain located in the inner mitochondrial membrane pumps protons from the mitochondrial matrix to the intermembrane space. Proton backflux from the intermembrane space to the matrix through the FoF1 ATPase leads to ATP synthesis.

Nucleomodulins: An emerging class of secreted bacterial effectors that enter the nucleus of the host cell, where they can affect various functions including DNA replication and repair, transcription, chromatin remodelling or RNA splicing [69].

Organelle: A cellular compartment that is separated from the cytosol by one or several membranes. Note that the definition of organelles may vary, from a stricter version only encompassing DNA-containing compartments (namely, mitochondria and plastids), to a more relaxed terminology that would include non-membrane enclosed structures visible in microscopy such as nucleoli, centrioles, stress- or P-granules. For the purpose of clarity in this review, we opted for the most-conventional definition as a membrane-enclosed intracellular compartment.

Unfolded Protein Response: A cellular quality control and defence mechanism, which is activated upon disruption of the proteostatic balance in the ER. To compensate the loss of ER function, this pathway aims at reducing the accumulation of abnormally-folded proteins, by (i) decreasing protein synthesis and translocation into the ER; (ii) stimulating the production of ER protein chaperones, which assist protein folding; (iii) promoting the degradation of unfolded proteins in the cytoplasm. If these mechanisms are ineffective, cell death is triggered [101]. 


\section{Figures}

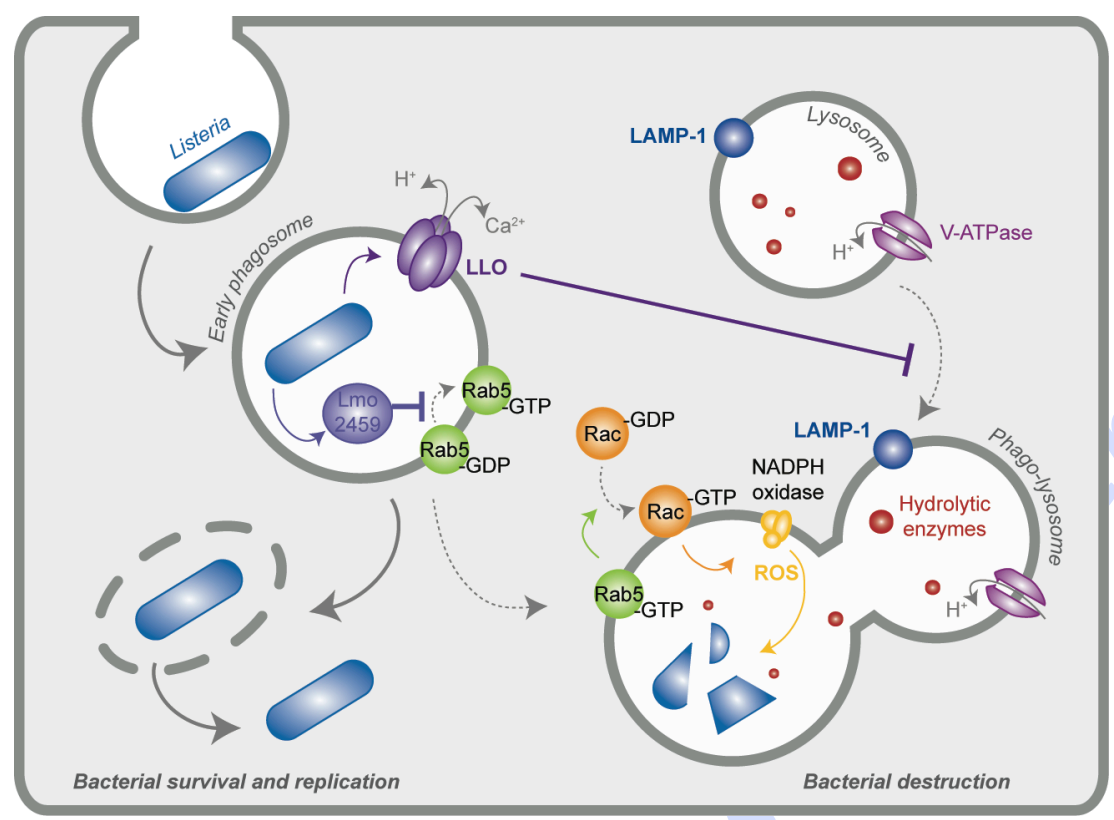

Fig. 1. Inhibition of phago-lysosomal maturation by Listeria. After phagocytosis by macrophages, the bacteria-containing phagosome may fuse with LAMP-1-positive lysosomes, into a phago-lysosome. ROS toxicity produced by NADPH oxidase and the action of hydrolytic enzymes, which is enhanced by the acidification of the organelle, results in bacterial killing and degradation. The secretion of LLO by Listeria decreases phagosomal calcium concentration and increases $\mathrm{pH}$, which impede phago-lysosomal fusion. Another secreted effector, Lmo2459, blocks the maturation of the phagosome via the inhibition of Rab5. 
(A)

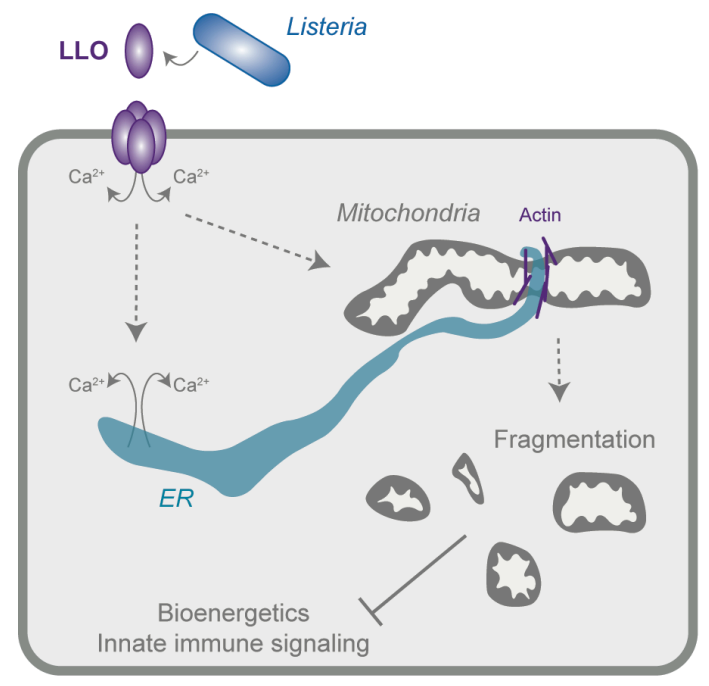

(B)

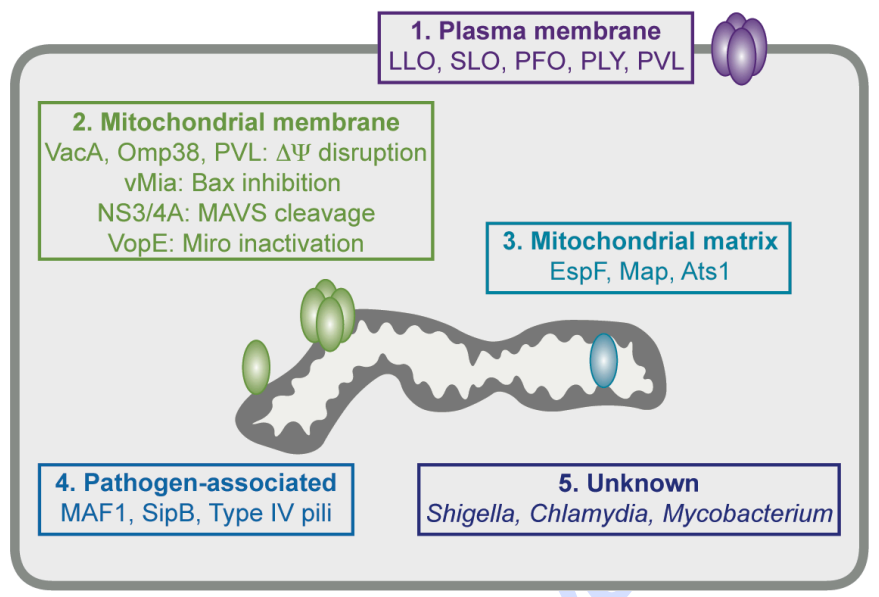

Fig. 2. Mitochondria and bacterial infections. (A) Listeria-induced mitochondrial fragmentation. LLO secreted by Listeria can form ion-permeable pores in the plasma membrane, thereby causing mitochondrial fragmentation. This fragmentation is facilitated by the ER and actin, but probably requires additional downstream factors, as it is independent of the main mitochondrial fission factor Drp1. Structural and functional disruption of the mitochondrial network causes a decrease in cellular bioenergetics and may modulate the mitochondria-based innate immune response. Although LLO has not been detected on the ER, it was shown to cause $\mathrm{IP}_{3} \mathrm{R}$-independent calcium release. (B) Pathogen strategies to interfere with host cell mitochondria. Bacterial, viral or protozoan proteins can target mitochondria in different ways. Several pore-forming toxins, such as Listeriolysin O (LLO, Listeria monocytogenes), Streptolysin O (SLO, Streptococcus pyogenes), Perfringolysin (PFO, Clostridium perfringens), Pneumolysin (PLY, Streptococcus pneumoniae) and PantonValentine leukocydin (PVL, Staphylococcus aureus) insert into the plasma membrane, thereby causing ion imbalances and activating signalling pathways that affect mitochondrial morphology and function (1). Other toxins, such as VacA (Helicobacter pylori), Omp38 (Neisseria gonorrhoeae) and PVL insert into the outer or inner mitochondrial membrane, causing a loss in the mitochondrial membrane potential $\Delta \Psi(2)$. The viral proteins vMia (cytomegalovirus) and NS3/4A (Hepatitis C) localize to the outer membrane and respectively induce Bax inhibition along with mitochondrial fragmentation, and MAVS cleavage. Vibrio cholerae VopE is a GTPase activating protein that inactivates Miro at mitochondria, inducing mitochondrial fragmentation. The secreted effectors EspF, Map (enteropathogenic Escherichia coli) and Ats1 (Anaplasma phagocytophilum) are imported into mitochondria and act "from within" to interfere with mitochondrial apoptosis pathways (3). Yet other effectors act on mitochondria while remaining pathogen-associated (4): Toxoplasma gondii MAF1 recruits mitochondria to the parasitophorous vacuole; Salmonella enterica SipB induces the formation of mitochondria-derived membrane structures; Neisseria gonorrhoeae type IV pili inhibit apoptosis. Other microbial effectors either remain unknown or act on mitochondria from unknown locations that include other organelles or the cytoplasm (5). 


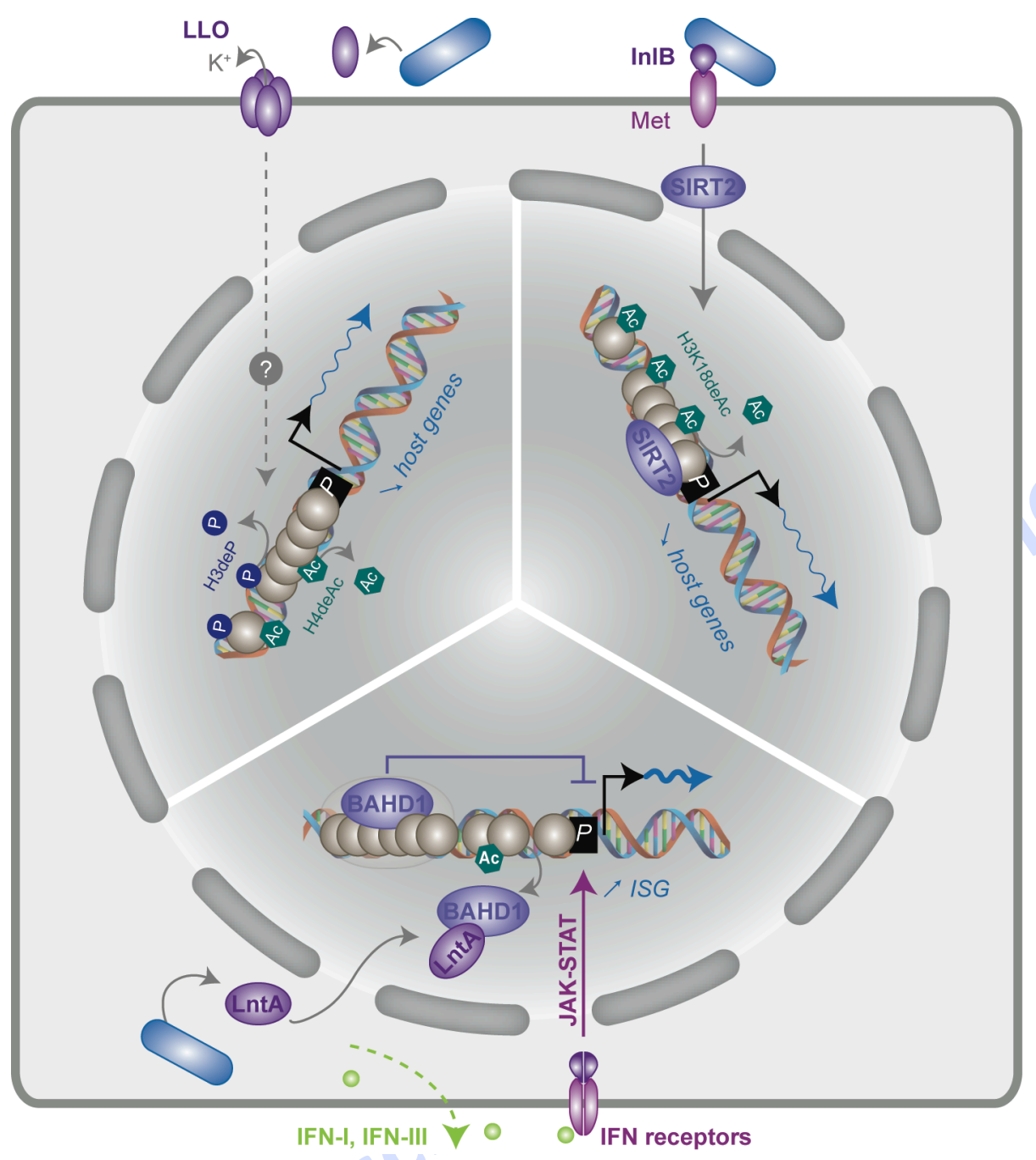

Fig. 3. Subversion of nuclear functions by Listeria. Left. By secreting the pore-forming toxin LLO, Listeria generates potassium efflux from the host-cell, which promotes a global deacetylation of histone $\mathrm{H} 4$ and dephosphorylation of Histone $\mathrm{H} 3$ on serine 10. This results in the down-regulation of a subset of host genes. Right. Binding of Listeria InlB to its receptor Met at the host cell membrane triggers, via a PI3K/Akt-dependent signalling cascade, the translocation of the lysine deacetylase SIRT2 to the nucleus. SIRT2 associates with chromatin at the promoter of various host genes, triggers the deacetylation of lysine 18 on $\mathrm{H} 3$, and thereby represses these genes. Bottom. In response to Listeria infection of epithelial cells, type I and III interferons are produced. However, the expression of ISGs is repressed downstream of JAK/STAT signalling, due to the condensation of a repressive complex at their promoter by the host chromatin component BAHD1. Under specific conditions, Listeria secretes the nucleomodulin LntA, which translocates to the nucleus, binds directly with BAHD1 and inhibits its function, resulting in an enhanced expression of ISGs. 